\title{
Prior Prophecy of Septicemia Through Machine Learning
}

\author{
Manikandan R a, Balaji S R b, Sakthivel R c , Gayathri L U d and Durga E d \\ a Professor, Dept of EIE,Panimalar Engineering College,India \\ ${ }^{b}$ Associate Professor, Dept of EIE,Panimalar Engineering College,India \\ ${ }^{c}$ Assistant Professor, Dept of EIE,Panimalar Engineering College,India \\ ${ }^{d}$ UG scholar, Dept. of EIE, Panimalar Engineering College,India.
}

\begin{abstract}
In recent days, diagnosis of sepsis involves a wide range of tests. Single test cannot tell that a person has sepsis. Undetected sepsis finally leads to death. The main drawback of sepsis is, there is much delay in predicting the diseases and identifying the various stages. Thus to avoid this delay in detection, we use Machine Learning concept. Thus by using this method prediction of sepsis has been increased and the mortality rate is reduced.
\end{abstract}

Keywords. Diagnosis, Severe sepsis, Septic shock, Machine Learning.

\section{Introduction}

Septicemia is a treacherous condition where it happens when an infant's or a normal person's immunity fights back against an infection. Sepsis develops when the chemicals of the immune system is released into the bloodstream to fight an infection which causes ${ }^{[8]}$ an inflammation throughout the body. Adults are mostly affected by sepsis, but infants are affected rapidly. Particularly newborns are more susceptible in developing sepsis [5] Generally infants who are extremely sick are cared by the specialist of the hospital in the ICU. Then many machines are used to monitor the body function such as heart rate, blood pressure and ventilator to help the child to breathe. Having any one symptom alone, does not develop Sepsis. When few of the mentioned symptoms happen together, then there is a possibility of sepsis infection. This disease dose than develop by the symptoms directly. The end results of many disease leads to Sepsis ${ }^{[1]}$.

\section{Existing system}

In existing system, the medical team diagnoses the symptoms of the disease from the patient's medical history, a physical exam and a test. Diagnosis will usually involve a wide range of tests. It depends on a positive blood test, blood culture, urine test, urine culture etc. It takes long time for prediction and the culture diagnosis ${ }^{[2]}$. It takes two to three days for the culture diagnosis result. However, it is unable to identify the severity of sepsis at instant. Because of this delay, the severity of the patient increases.

\footnotetext{
${ }^{1}$ Manikandan R, Professor, Department of EIE, Panimalar Engineering College, India Email: money_kandan2004@yahoo.co.in
} 
At present this type of early prediction has been carried out at Howard County General Hospital, Columbia, Maryland, USA ${ }^{[7]}$.

\subsection{Limitations of Existing System}

- In the existing system the data set is typically small.

- It provides low accuracy.

- More time consuming.

- Use of number of algorithm

\section{Problem Identification}

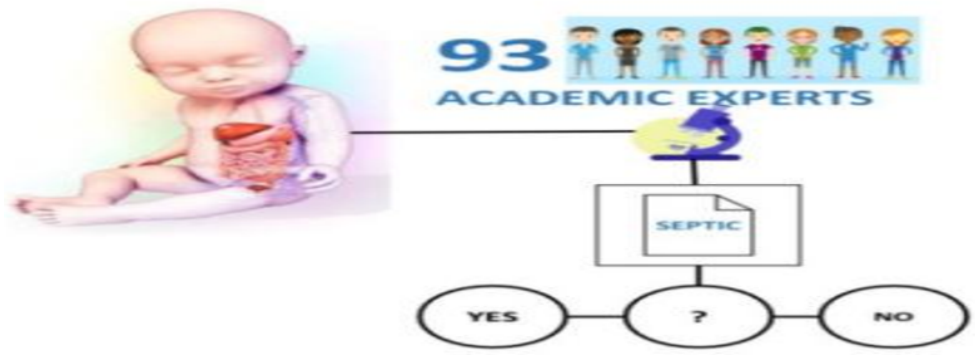

Figure 1. Many experts are required for diagnosis

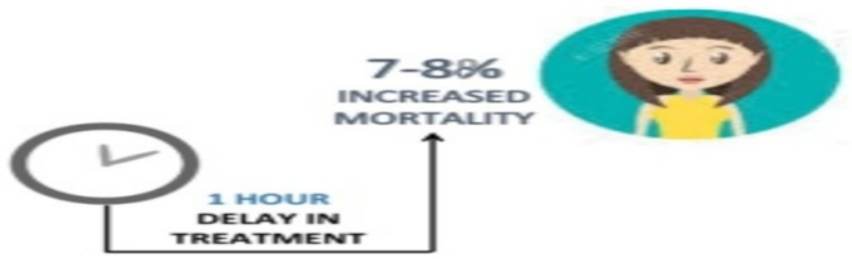

Figure 2. Increased Mortality Statistics

The main drawback is that there is much delay in predicting the diseases and identifying the various stages. Usually this is done through culture test. It may at least take two to three days to get the result. By the time the organs starts to dysfunction ${ }^{[4]}$. It must be treated very fast. As every hour when Sepsis is not treated it increases the death rate ${ }^{[9]}$.

\section{Proposed system}

To overcome the delay in predicting the disease and identifying the stages, this is done through culture test. It may at least take two to three days to get the result. By the time the organs starts to dysfunction.Thus to avoid this delay we use Machine Learning concept. Thus by implementing this project two third of the patients were saved before their organs starts to fail. Thus by using this method, the performance of prediction has been increased and the mortality rate can be reduced. The data set used for sepsis prediction has been collected from Rajah Muthaih Medical College Hospital, Annamalai University, Anna malai Nagar, Chidambaram-608002, Tamil Nadu. Here 
only one algorithm has been used for training, predicting and validating the model. The technique used for prediction is predictive analytics. This technique is used to predict on unknown future events. It uses many techniques such as Statistics, Machine Learning, Artificial Intelligence, etc to analyze the current data with the dataset to make predictions.
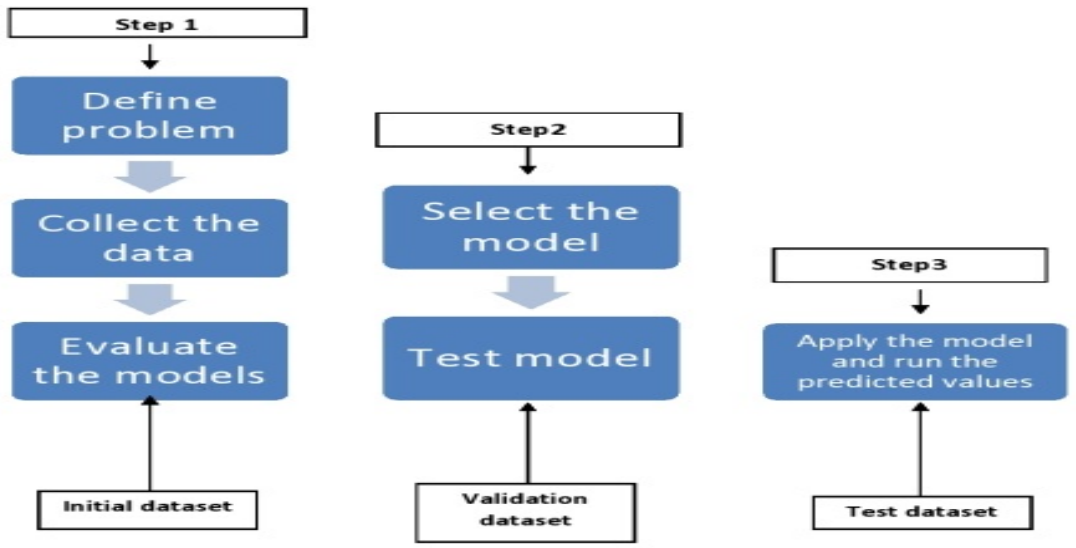

Figure 3. Predictive Analytics Model

\section{Model description}

The clinical data set used in this paper is the real-life hospital data. The objective of this study is to predict whether the patient has the chance of sepsis or not, from the medical history. In this model we divide the data set into training and testing data set. The algorithm used for this model is a Random Forest algorithm

\section{Methodology}

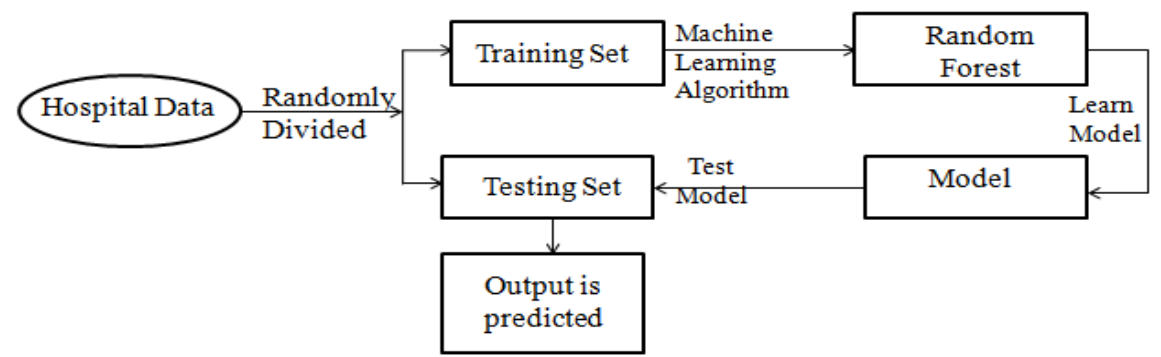

Figure 4. Proposed system Block Diagram

The sample data consists of about one lakh entries which constitutes sepsis patient's records and the normal records of a person who has no sepsis. The parameters that we have collected for sepsis detection are Respiratory rate, Heart rate, WBC count, Temperature, Urine test result, SpO2, Systolic \& Diastolic pressure, Glucose, Creatinine, Bilirubin, INR (International Normalised Ratio), Platelets etc. Training and testing of Data set is an important part of evaluating the data. In this model we 
have taken $70 \%$ of data for training and $30 \%$ of data for testing the model. Training data includes both input data and the corresponding expected output. Testing data includes only input data, not the corresponding expected output.

\subsection{Algorithm description}

"Many trees together make a forest". Likewise many decision trees together which is chosen randomly referred as Random Forests or Random Decision. It is easy to use a Machine Learning algorithm. Learning method operates by constructing multiples of decision trees at training time and at output time. In general, the more trees in the forest the more strong the forest looks. Likewise higher the number of trees in the forest gives the high accuracy results.

\subsection{Testing and training model}

The process of training a model involves providing a learning algorithm with training data to learn. The training data must contain the correct answer, which is known as an attribute. The learning algorithm finds patterns in the training data that map the input data attributes to the target (the answer that we want to predict) and it outputs an Machine Learning model that captures these patterns.From the dataset the machine takes the highest and lowest value of each parameter and calculates its average value. Then the machine compares the average value with the normal range of the parameter. If it matches with the normal value then there is no disease predicted. But if the value does not match the condition then there is a existence of disease. This is how the machine predicts the disease.

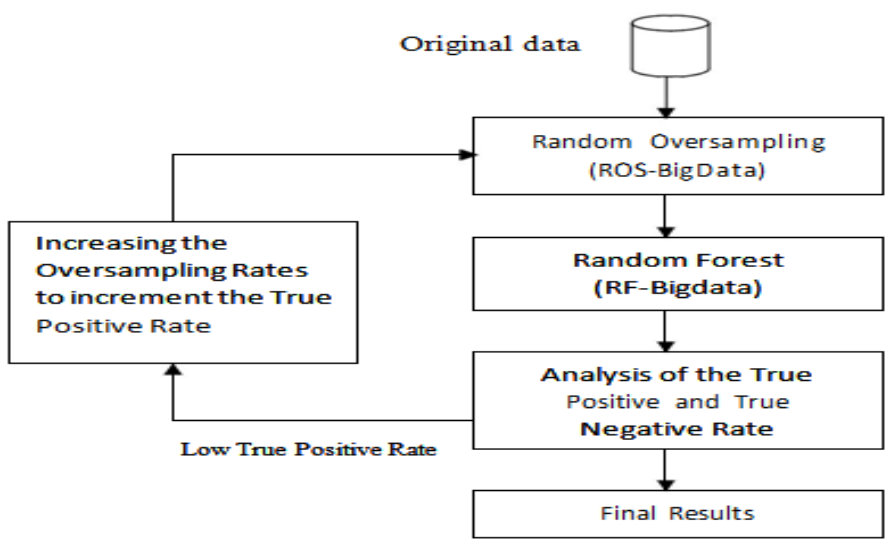

Figure 5. Random Forest Algorithm Flowchart

\section{Results and discussion}

Thus we have created a Machine Learning model for the prediction of septicemia disease. The prediction model consists of three stages. They are prediction of sepsis, severe sepsis and septic shock. These are the five stages which involves the sepsis prediction. The fifth stage - improve, is the stage where we have developed the sepsis prediction. 


\subsection{Overview of sepsis prediction}

Stage 1: Get the data :All the clinical data has been collected from the Rajah Muthaih Medical College Hospital, Annamalai University, Annamalai Nagar-608002, Tamil Nadu.

Stage2: Clean, prepare and manipulate data :The clinical data which has been collected are prepared and manipulated according to the model, which is going to be trained for sepsis prediction.

Stage3: Train the model :The prepared and manipulated data has been trained with the help of the Machine Learning algorithm. In this project we have used Random Forest algorithm, which combines all the functions of classification, regression and clustering algorithm.

Stage4: Test the data :In this stage, the trained model is tested by feeding the input to the model. The given input is analyzed with the clinical dataset and the result is interpreted.

Stage 5: Improve: This is the final stage where we obtain the output. In this project we interpret the output in three stages namely sepsis, severe sepsis and septic shock.

Table 1. Result analysis

\begin{tabular}{|c|c|}
\hline Stage of sepsis & Septic shock \\
\hline Heart rate & 140 \\
\hline Respiratory Rate & 76 \\
\hline SpO2 & $96 \%$ \\
\hline Urea & 53 \\
\hline Creatinine & 1.5 \\
\hline CRP & 4.5 \\
\hline Random Blood Sug & 45 \\
\hline White Blood Cells & 20.3 \\
\hline Presence of disease & Yes \\
\hline Stage of septicemia & sepsis \\
\hline
\end{tabular}

\subsection{Output of proposed system}

First stage output: First the symptoms of sepsis such as Fever and chills, low body temperature, expeditious pulse rate, expeditious breathing, Nausea and vomiting etcare detected. The clinical parameters used to detect the symptoms are respiratory 
rate, heart rate, WBC count. After the symptoms have been detected, first stage output that is sepsis is detected.

Second stage output: The clinical parameters used to detect the severe sepsis are $\mathrm{SpO} 2$ and urine test result. After entering the parameters the trained model analyze with the dataset and then the second stage output that is severe sepsis can be detected.

Third stage output: The clinical parameters used to detect the severe sepsis are urine test result, systolic and diastolic pressure, glucose, Creatinine, lactate, Bilirubin rate, INR ratio and platelets rate. After entering the parameters the trained model analyze with the dataset and then the third stage output that is septic shock can be detected.

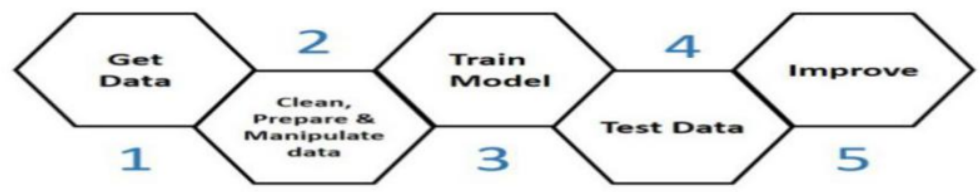

Figure 6. Process flow diagram of Sepsis Prediction

Thus by implementing this project two third of the patients were saved before the organs starts to fail. Hence this method of performance prediction has been increased and the mortality rate can be reduced.Compared to existing system the mortality rate in proposed system has been reduced to $60 \%$. Hence the accuracy has been increased.Existing work focuses on many algorithms ${ }^{[6]}$ for predicting the disease. But in this project we use only one algorithm for prediction. Compared to several typical prediction algorithms, the proposed algorithm is time consuming. In future, with help of this Machine Learning technology, prediction of the disease can be enhanced. After prediction, by recognizing the severity the machine can even prescribe medicine for the patients. This type of projects will be much useful to the hospital management system.If the output of the project turns out positive for existence of sepsis, it is advised to consult a doctor before taking further remedy or action. Since this project is only used for prediction and not detection of sepsis.

\section{References}

[1] Disease prediction by Machine Learning over Bigdata from Healthcare communities.jpinfotech projects 25 October 2017.

[2] R. Shiva Shankar, M. Mounica Devi, J. Rajanikanth and G. Mahesh. An Efficient Algorithm for Multimodal Epidemic Liability Prediction over Big. Data International journal of Pure and Applied Mathematics Volume 1192018.

[3] Dr. D. Suresh Kumar. Sepsis and sepsis Mimics.Sepsis update Article from Appollo Hospitals, Chennai 2013 to 2017.

[4] Sylvia Owusu-Ansah.Sepsis in Infants and Children - HealthyChildren. Orgtrusted by American Academy of Pediarics. 9 November 2017.

[5] Sepsis and Children.Article in Sepsis alliance 13 December 2017.

[6] D K Harini, M Natesh. Prediction of probalility of disease based on symptoms using Machine Learning Algorithm International Research Journal of Engineering and Technology IRJET. Bioinformatics and Biomedical Engieering $5^{\text {th }}$ International Work-Conference Volume: 05 April 26-28 2017. 
[7] Hoyt Burdick, Eduardo Pino, Denise Gabel-Comeau, Carol Gu, Heidi Huang, Anna Lynn-Palevsky, Ritankar Das Evaluatig a sepsis prediction machine learning algorithm using minimal electronic health record data in the emergency depeartment and intensive care unit before and after comparative study 28 November 2018.

[8] S.Hema Kumar, J.Uday Kiran, V.D.Ambeth Kumar, G.Saranya, Ramalakshmi V, "Effective Online Medical Appointment System”, International Journal of Scientific \& Technology Research, Volume 8, Issue 09, September 2019, Pages $803-805$. 